\title{
Digital Image Colorization Based on Probabilistic Distance Transform
}

\author{
Przemyslaw Lagodzinski ${ }^{1}$ and Bogdan Smolka ${ }^{2}$ \\ Silesian University of Technology, \\ Akademicka 16, 44-100 Gliwice, Poland \\ ${ }^{1}$ Institute of Computer Science \\ ${ }^{2}$ Institute of Automatic Control \\ \{przemyslaw.lagodzinski, bogdan.smolka\} @polsl.pl
}

\begin{abstract}
Colorization denotes a process of adding color to gray-scale images, movies and TV programs. This process replaces the intensity value of an image pixel with a vector in a three dimensional color space composed of hue, saturation and luminance, and since this mapping between intensity and color, has no inherently 'correct solution', human interaction and external information usually plays a large role. In this paper we present a novel colorization method that takes advantage of the modified morphological distance transform to automatically propagate the color scribbled by a user on the gray-scale image. The introduced modification of the distance transform is based on the Gibbs distribution which governs the behavior of a virtual particle performing a random walk on the image domain. Such a modification allows for the application of the distance transform to the gray-scale images and yields high quality colorization results.
\end{abstract}

Keywords: Colorization, distance transform, computer vision.

\section{Introduction}

With the rapid development of computer technology, adding color to the grayscale images and movies in a way that looks natural to human observers became a problem that challenged the motion picture industry as well as computer vision community. Despite the fact that colors increase the visual appeal of an image, colorization aroused many controversies especially with reference to the work of art and classic movie films. Nevertheless, in the last few years, several advanced and effective techniques have been proposed. These techniques are based on: luminance keying and color transfer [1], image analogies [2], motion estimation [3], segmentation [4], color prediction [5], probabilistic relaxation [6] and chrominance blending [7, among many other techniques.

In this paper we show that using our novel colorization method based on the modified distance transformation, it is possible to obtain satisfactory colorization results in a very short time and with small amount of work.

This paper is organized as follows. The next section presents the proposed colorization algorithm. Subsection 2.1 reviews a standard fast algorithm for computing the distance transform (DT). In subsection 2.2 we extend the distance

J. Ruiz-Shulcloper and W.G. Kropatsch (Eds.): CIARP 2008, LNCS 5197, pp. 62666342008.

(C) Springer-Verlag Berlin Heidelberg 2008 
transform using a probabilistic approach and introduce a correction factor for additive color mixing process in order to preserve original pixels luminance. Finally, in Section 3 we present our colorization results and in Section 4 we formulate the final conclusions.

\section{The Proposed Colorization Algorithm}

In this section we present our novel algorithm for image colorization that exploits the probabilistic distance transformation (DT). Our goal is to create a fast and effective colorization algorithm which does not require precise segmentation as well as any other color images as a reference (see Fig. 1).
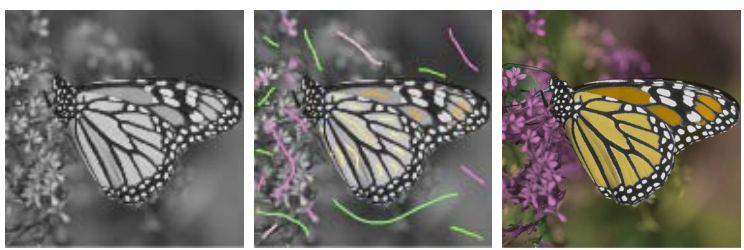

Fig. 1. Illustration of the proposed colorization process: original gray-scale image (left), image scribbled with color (middle), and our colorization result (right)

\subsection{Distance Transform}

Since the color is provided simply by scribbling the image, the first step of our algorithm, after the user inserts the scribbles, is to isolate them and compute their distance to all the pixels of the source gray-scale image. We use the approximation of the Euclidean distance in order to significantly decrease the computational load of the DT algorithm [8].
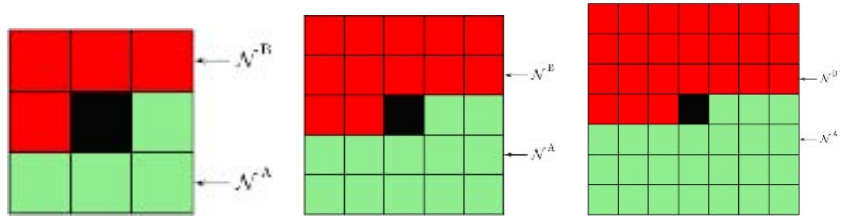

Fig. 2. $\mathcal{N}^{B}$ (red) and $\mathcal{N}^{A}$ (green) neighbors of the central pixel (black) within the structuring element of size: $3 \times 3,5 \times 5$ and $7 \times 7$

Thus, let $\mathcal{P}$ be a binary image defined on the image domain $\mathcal{G}$, where:

$$
\langle\mathcal{P}\rangle=\{p: \mathcal{P}(p)=1\},\langle\overline{\mathcal{P}}\rangle=\{p: \mathcal{P}(p)=0\},
$$

are proper subsets of $\mathcal{G}$. For any metric, the distance transform associates with every pixel $p$ of $\langle\overline{\mathcal{P}}\rangle$ the distance from $p$ to $\langle\mathcal{P}\rangle$. 
For any $p \in \mathcal{G}$ let $\mathcal{N}_{p}^{B}$ (pixels in the neighborhood relation before scan) be the set of pixels adjacent to $p$ that proceeds $q$ when $\mathcal{G}$ is scanned, and let $\mathcal{N}_{p}^{A}$ (after scan) be the remaining neighbors of $p$, (see Fig. 2). Then during the first scan (in top-left to bottom-right direction) we compute:

$$
\left\{\begin{array}{cll}
f_{1}(p)=0 & : & p \in\langle\mathcal{P}\rangle, \\
\min \left\{f_{1}(q)+1: q \in \mathcal{N}_{p}^{B}\right\}: & p \in\langle\overline{\mathcal{P}}\rangle .
\end{array}\right.
$$

After the first scan, we perform the second scan in the opposite direction (bottom right to top-left), and compute the following:

$$
f_{2}(p)=\min \left\{f_{1}(p), f_{2}(q)+1: q \in \mathcal{N}_{p}^{A}\right\} .
$$

Thus, after the second scan we obtain the distance values that can be expressed as intensities of points within the gray-scale image as sown in Fig. 3 ,
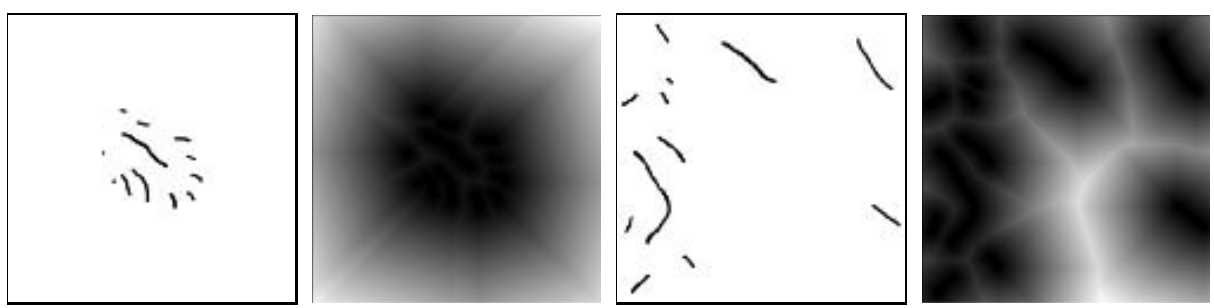

Fig. 3. Isolated yellow and pink scribbles from Fig. 1 and the distance transform 8 performed on those scribbles

\subsection{Probabilistic Distance Transform}

The modified distance transform, which can be considered as a revision to [9], is based on a model of a virtual particle, which performs a random walk on the image lattice. It is assumed, that the probability of a transition of the walking particle from a lattice point to a point belonging to its neighborhood is determined by the Gibbs statistical distribution. Thus, the probability $P_{i j}$ of a jump from the current site $i$ to site $j$ is dependent on the local environment and is calculated as 10]:

$$
P_{i j}=\frac{\exp \left\{-\frac{E_{i j}}{k T}\right\}}{Z_{i j}}, \quad Z_{i j}=\sum_{j \in W_{i}} \exp \left\{-\frac{E_{i j}}{k T}\right\},
$$

where $W_{i}$ denotes the filtering window centered at site $i, E_{i j}$ is the energy barrier between the sites $i$ and $j, k$ is the Boltzmann constant and $T$ denotes the temperature of the statistical ensemble. 

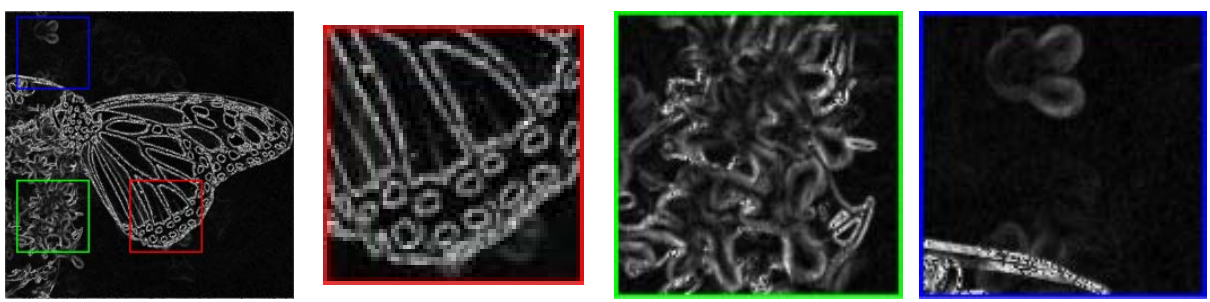

Fig. 4. Illustration of the measure $D_{i}^{\mathcal{N}}$ on a gray-scale image depicted in Fig. 1

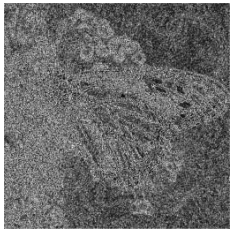

(a) $\beta=2$

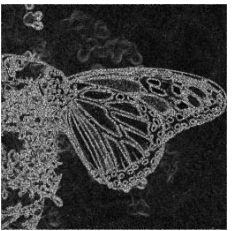

(b) $\beta=6$

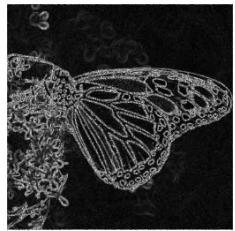

(c) $\beta=10$

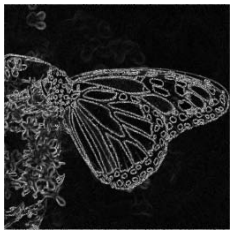

(d) $\beta=14$

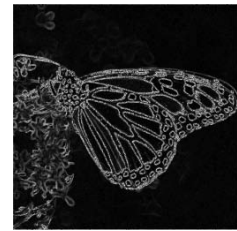

(e) $\beta=18$

Fig. 5. Local image homogeneity $D_{i}^{\mathcal{N}}$ dependence on the $\beta$ parameter

Let us define the energy barrier between the pixel sites $i$ and $j$ as $E_{i j}=$ $\left|Y_{i}-Y_{j}\right|$, where $Y_{j}$ denotes the pixel's intensity at a position $j$. Setting $k T=\beta$ we obtain:

$$
P_{i j}=\frac{1}{Z_{i j}} \exp \left\{-\frac{\left|Y_{i}-Y_{j}\right|}{\beta}\right\} .
$$

The probability that a virtual particle will remain at the current site is:

$$
P_{i i}=\frac{1}{Z_{i i}}=\left(\sum_{j \in W_{i}} \exp \left\{-\frac{\left|Y_{i}-Y_{j}\right|}{\beta}\right\}\right)^{-1}=\left(1+\sum_{j \in \mathcal{N}_{i}} \exp \left\{-\frac{\left|Y_{i}-Y_{j}\right|}{\beta}\right\}\right)^{-1}
$$

where $\mathcal{N}_{i}$ denotes the pixels which are in neighborhood relation with the pixel at site $i$.

This probability can be seen as a measure of the homogeneity of the local image structures, as it takes low values in the smooth image regions and attains large values in the vicinity of edges. In order to evaluate the smoothness of the local neighborhood of a pixel at position $i$ we can define a measure $D_{i}^{\mathcal{N}}=$ $\sum_{j \in \mathcal{N}_{i}} P_{j j}$, which provides the information about the local image homogeneity in the neighborhood $\mathcal{N}$ of the pixels at site $i$. In this way:

$$
D_{i}^{\mathcal{N}}=\sum_{j \in \mathcal{N}_{i}}\left(1+\sum_{l \in \mathcal{N}_{j}} \exp \left\{-\frac{\left|Y_{j}-Y_{l}\right|}{\beta}\right\}\right)^{-1}
$$




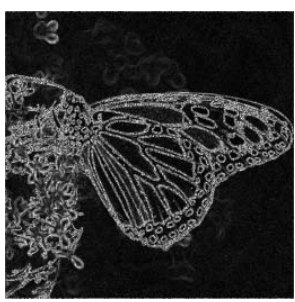

(a) $3 \times 3$

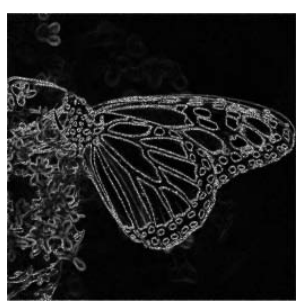

(b) $5 \times 5$

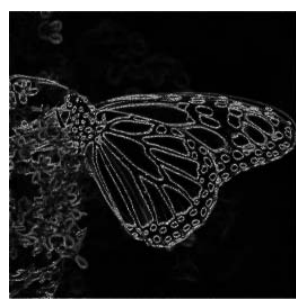

(c) $7 \times 7$

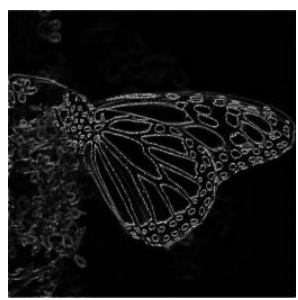

(d) $9 \times 9$

Fig. 6. Influence of the structuring element size on local image homogeneity $D_{i}^{\mathcal{N}}$

and the Eqs. (2) and (3) can be modified as

$$
\begin{cases}f_{1}(p)=0 & : p \in\langle\mathcal{P}\rangle \\ \min \left\{f_{1}(q)+D_{p}^{B}: q \in \mathcal{N}_{p}^{B}\right\} & : p \in\langle\overline{\mathcal{P}}\rangle \\ f_{2}(p)=\min \left\{f_{1}(p), f_{2}(q)+D_{p}^{A}\right\}: q \in \mathcal{N}_{p}^{A}\end{cases}
$$

where $D_{p}^{B}$ and $D_{p}^{A}$ denote the sum of probabilities calculated according to Eq. (7), where $B$ and $A$ denote the set of pixels from the neighborhood before and after the scan, (Fig. 2).

The measure $D_{i}^{\mathcal{N}}$ calculated for the image in Fig. 1 is visualized in Fig. 4 . The $\beta$ parameter in Eq. (77) influences significantly the local image homogeneity as depicted in Fig. 5. Also the number of $\mathcal{N}^{B}$ and $\mathcal{N}^{A}$ neighbors contained within the structuring element of a different size exerts a strong influence on the local image homogeneity map as shown in Fig. 6.

In order to use the values of the probabilistic DT as weights in the blending of colors originating from successive scribbles, we introduced a kernel function transforming their values: $F_{G}=\exp \left\{-(d / h)^{2}\right\}$, where the parameter $h$, set by a user, determines the smoothness of the Gaussian function and $d$ denotes the value of the extended DT we obtain from Eq. (8) for each point of the gray-scale image, (see Fig. 7).

The values of the kernel function are then used as weights to determine the color $C$ of a given point $p$ during the additive color mixing process defined by:

$$
C(p)=\frac{C_{1} \cdot F_{1}(d, p)+C_{2} \cdot F_{2}(d, p)}{F_{1}(d, p)+F_{2}(d, p)} \cdot r(p),
$$
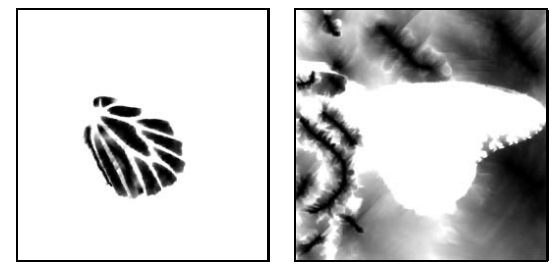

Fig. 7. Probabilistic distance transform performed on the isolated yellow (left) and pink scribbles (right) from the gray-scale in Fig.1 

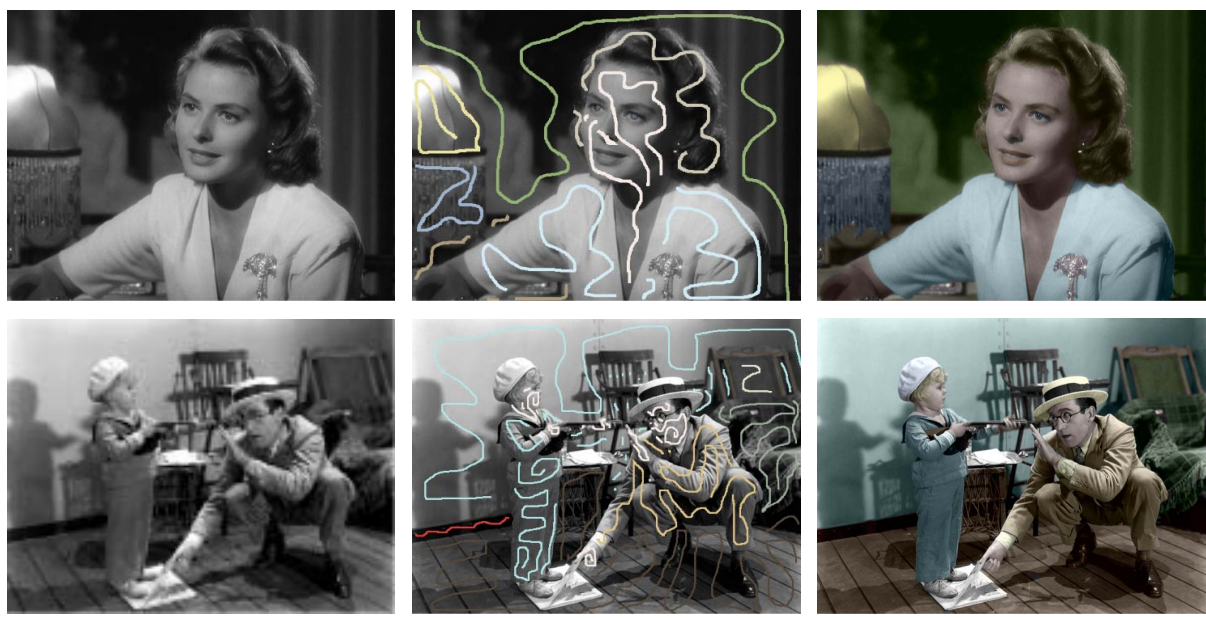

Fig. 8. Colorization of frames from the movie Casablanca and a movie with Harold Lloyd. In columns: gray-scale images taken from www.doctormacro.info, images scribbled with color and our colorization result.
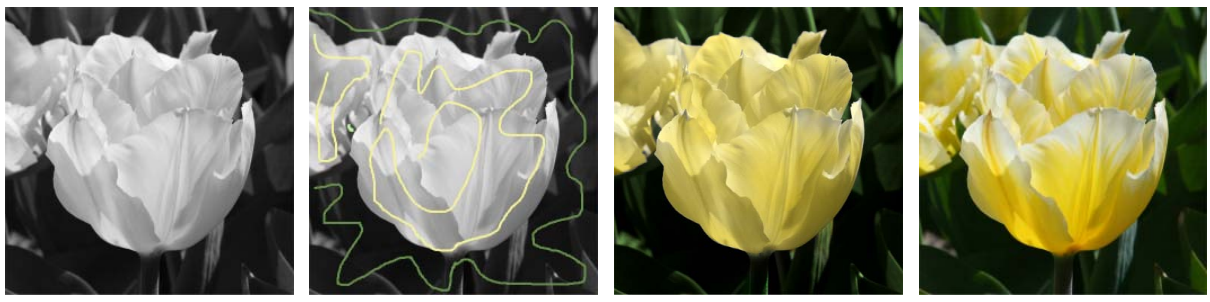

Fig. 9. Example of natural image colorization. From the left: gray-scale image, scribbled with color, our colorization result, and original color image for comparison.
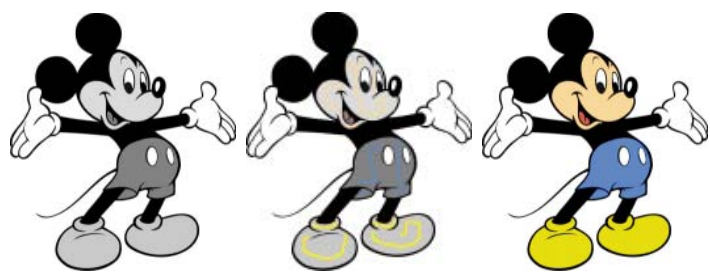

Fig. 10. Cartoon colorization example. From the left: gray-scale image, scribbled with color and colorization result.

where $C_{1}$ and $C_{2}$ are colors scribbled by the user and $F_{1}(d, p), F_{2}(d, p)$ are weights obtained for a given point $p$ using the Gaussian kernel function. The $r(p)$ is a correction factor which allows us to preserve the original intensity $\mathcal{Y}(p)$ from the source gray-scale image in newly propagated color: 


$$
r(p)=\frac{\mathcal{Y}(p)}{\max \left\{C_{1} \cdot F_{1}(d, p), C_{2} \cdot F_{2}(d, p)\right\}} .
$$

Since the proposed algorithm is iterative and colors are added one by one, indices 1 and 2 in Eq. (9) and (10) correspond respectively to the current and previous colorization step.
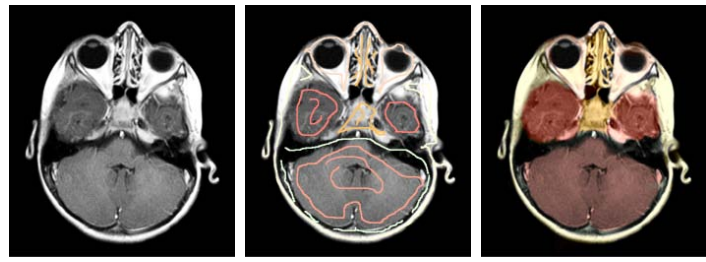

Fig. 11. MRI image colorization examples. From the left: gray-scale image, image scribbled with color and the colorization result.

\section{Results}

The results shown here were obtained using the presented method that works on the basis of the probabilistic distance transformation. The proposed solution is iterative and adds color one by one, (8-11). As can be observed, the proposed technique can be successfully applied to the colorization of historical photographs, cartoons and also medical images.

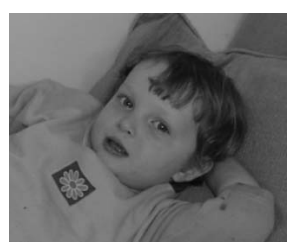

(a) source image

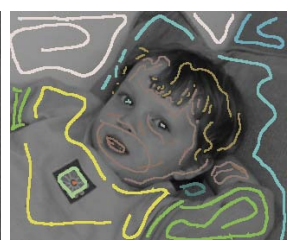

(b) scribbled image

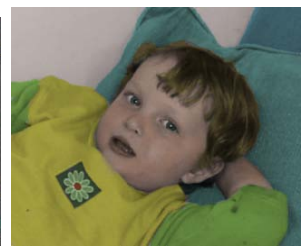

(c) our result

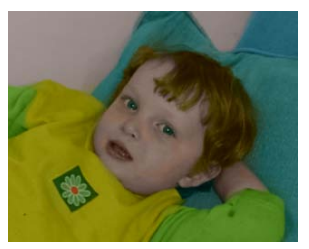

(d) Levin's result, , 4]

Fig. 12. Comparison of the proposed method with the algorithm described in [4: (a) source gray-scale image, (b) image with color scribbles, (c) our colorization result, (d) result of colorization obtained using the method proposed in 4]

In [11, the authors presented the comparison of the processing time between their algorithm and the method proposed in [4. Taking the same set of test images our algorithm is much faster and produces the computation complexity results summarized in Tab. 1 .

Although the code is not fully optimized, this implementation of our algorithm works fast enough to allow the user for interactive work without noticeable delays 
Table 1. Comparison of the processing time of colorization methods

\begin{tabular}{|c|c|c|c|c|}
\hline & Image size & Method in [4] & Method in [1] & Proposed \\
\hline \hline Cats & $319 \times 267$ & $15.20 \mathrm{~s}$ & $0.71 \mathrm{~s}$ & $0.34 \mathrm{~s}$ \\
\hline Girl & $318 \times 238$ & $10.12 \mathrm{~s}$ & $0.42 \mathrm{~s}$ & $0.36 \mathrm{~s}$ \\
\hline Building & $399 \times 299$ & $15.26 \mathrm{~s}$ & $0.91 \mathrm{~s}$ & $0.58 \mathrm{~s}$ \\
\hline
\end{tabular}

and achieving real-time preview. A comparison with the method presented in [4] shown in Fig. 12 confirms the good performance of the novel colorization method.

The future work will focus on further decrease of the computational load of the algorith and on the automatic adjustment of the smoothing parameter of the kernel function, which should automatically adjust to the image structures.

\section{Conclusions}

In the paper a new fast colorization technique based on a novel extension of the distance transform has been presented. The new distance transform is based on the Gibbs distribution which describes the behavior of a virtual particle performing a random walk on the image domain. The results of experiments performed on various gray-scale images show that the proposed approach yield good results and can be applied for the purposes of the virtual restoration of old movies, cartoons, medical images and works of art. In the future work, we will work on the optimization of the code of our application, which will further shorten the time of colorization process in the case of very large images.

\section{References}

1. Reinhard, E., Ashikhmin, M., Gooch, B., Shirley, P.: Color Transfer Between Images. IEEE Computer Graphics and Applications 21(5), 34-41 (2001)

2. Welsh, T., Ashikhmin, M., Mueller, K.: Transferring Color to Grayscale Images. In: Proceedings of ACM SIGGRAPH Conference, pp. 277-280 (2002)

3. Pan, Z., Dong, Z., Zang, M.: A New Algorithm for Adding Color to Video or Animation Clips. In: Proceedings of WSCG - Int. Conference in Central Europe on Computer Graphics, Visualization and Computer Vision, pp. 515-519 (2004)

4. Levin, A., Lischinski, D., Weiss, Y.: Colorization Using Optimization. In: Proceedings of ACM SIGGRAPH Conference, pp. 689-694 (2004)

5. Madeira, J.S., Stork, A., Grob, M.H.: An Approach to Computer-Supported Cartooning. The Visual Computer 12, 1-17 (1996)

6. Horiuchi, T.: Estimation of Color for Gray-level Image by Probabilistic Relaxation. In: Proceedings of IEEE International Conference on Pattern Recognition, pp. 867870 (2002)

7. Yatziv, L., Sapiro, G.: Fast Image Video Colorization Using Chrominance Blending. IEEE Transactions on Image Processing 15(5), 1120-1129 (2006)

8. Klette, R., Rosenfeld, A.: Digital Geometry - Geometric Methods for Digital Picture Analysis. Morgan Kauffman, San Francisco (2004) 
9. Lagodzinski, P., Smolka, B.: Fast Digital Image Colorization Technique. In: IEEE International Symposium on Signal Processing and Information Technology, pp. 813-818 (2007)

10. Smolka, B., Wojciechowski, K.W.: Random Walk Approach to Image Enhancement. Signal Processing 81(3), 465-482 (2001)

11. Li, Y., Lizhuang, M., Di, W.: Fast Colorization Using Edge and Gradient Constraints. In: Proceedings of the 15th International Conference in Central Europe on Computer Graphics, Visualization and Computer Vision, pp. 309-316 (2007) 